\section{UJMM

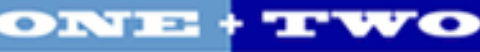

Volume 8 | 2017 Fall 2017

\section{Undergraduate Journal of Mathematical} Modeling: One + Two

2017

\title{
Analysis of Rainfall in Tampa
}

Amy Polen

University of South Florida

Advisors:

Arcadii Grinshpan, Mathematics and Statistics

Jennifer Collins, Geography, Environment, and Planning

Problem Suggested By: Amy Polen

Follow this and additional works at: https://digitalcommons.usf.edu/ujmm

Part of the Mathematics Commons

UJMM is an open access journal, free to authors and readers, and relies on your support:

Donate Now

\section{Recommended Citation}

Polen, Amy (2017) "Analysis of Rainfall in Tampa," Undergraduate Journal of Mathematical Modeling: One + Two: Vol. 8: Iss. 1, Article 4.

DOI: http://doi.org/10.5038/2326-3652.8.1.4885

Available at: https://digitalcommons.usf.edu/ujmm/vol8/iss1/4 


\title{
Analysis of Rainfall in Tampa
}

\begin{abstract}
Rainfall for a region is very important to define, because it affects an ecosystem health, wildlife, and even human behavior. Using data obtained from National Oceanographic and Atmospheric Administration (NOAA) at the Tampa Bay International Airport and graphing it, the total rainfall for a year was estimated by both a rough trapezoidal Riemann's sum approximation and a definite integral generated by polynomial regression. It was determined that both approximations gave an underestimate of the total rainfall that was measured, but the polynomial integral gave a reasonable estimate with a small percent error. It was seen through the graphical representation of this data and the overall recorded total rainfall that indeed it did match the humid subtropical climate type of Tampa, with a spike in rainfall in the summer and precipitation amount that matched the Köppen climate classification system for this climate type. In guiding future measurements of rain it is useful to use a trendline to predict rainfall over a given period of time.
\end{abstract}

\section{Keywords}

rainfall analysis, Köppen climate classification system, Riemann's sum, polynomial approximation

\section{Creative Commons License}

(c) (i) (9)

This work is licensed under a Creative Commons Attribution-Noncommercial-Share Alike 4.0 License. 


\section{PROBLEM STATEMENT}

The amount of rain one area receives can affect many different aspects of the environment, such as river discharge, biome diversity, and overall ecosystem health. Also it can influence human population, such as the amount of water in freshwater reserves like the Floridian Aquifer, flooding, and sinkholes. By employing a trapezoidal Riemann's sum and a polynomial integral the total rainfall at Tampa International Airport can be estimated for an entire calendar year and observed for accuracy against the total amount measured by NOAA. This will be compared with the Köppen climate classification to see if this past year's precipitation matches the climate classification for the Tampa Bay region.

\section{MOTIVATION}

The Köppen-Geiger climate classification system is the most commonly system used worldwide to describe the climate of a region, with five major categories: tropical moist, dry, moist mid-latitude with cold winters, moist mid-latitude with mild winters, and polar climates (Fundamentals eBook, n.d.). Tampa falls into the category of humid subtropical climate (Cfa, Figure 1). It implies dry winters and mild winters with a peak in rainfall during the summer. 


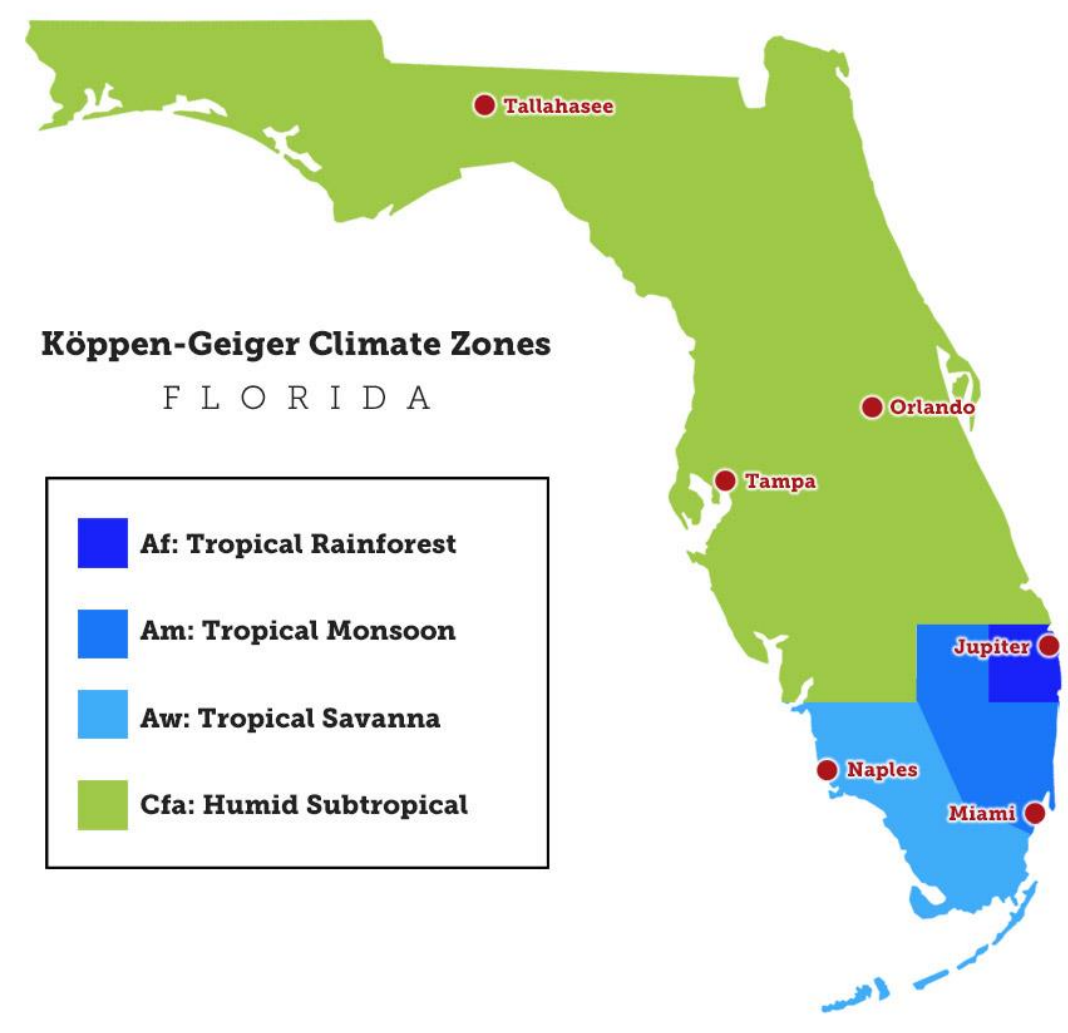

Figure 1: Florida Climate Zones (WeatherStem)

Regional rainfall is important for a variety of reasons. From an environmental standpoint, rain is necessary for many mid-latitude and tropical climates where it plays a vital role in ecosystems. In Florida, rain is necessary for indigenous wildlife such as alligators or panthers. Also it is necessary for the flora which is quite affected by drought. This removal of native species which rely on water can lead to the dominance of other species such as cabbage palms (Figure 2), which are quite fire-resistant, and invasive species such as the Brazilian pepper tree. 


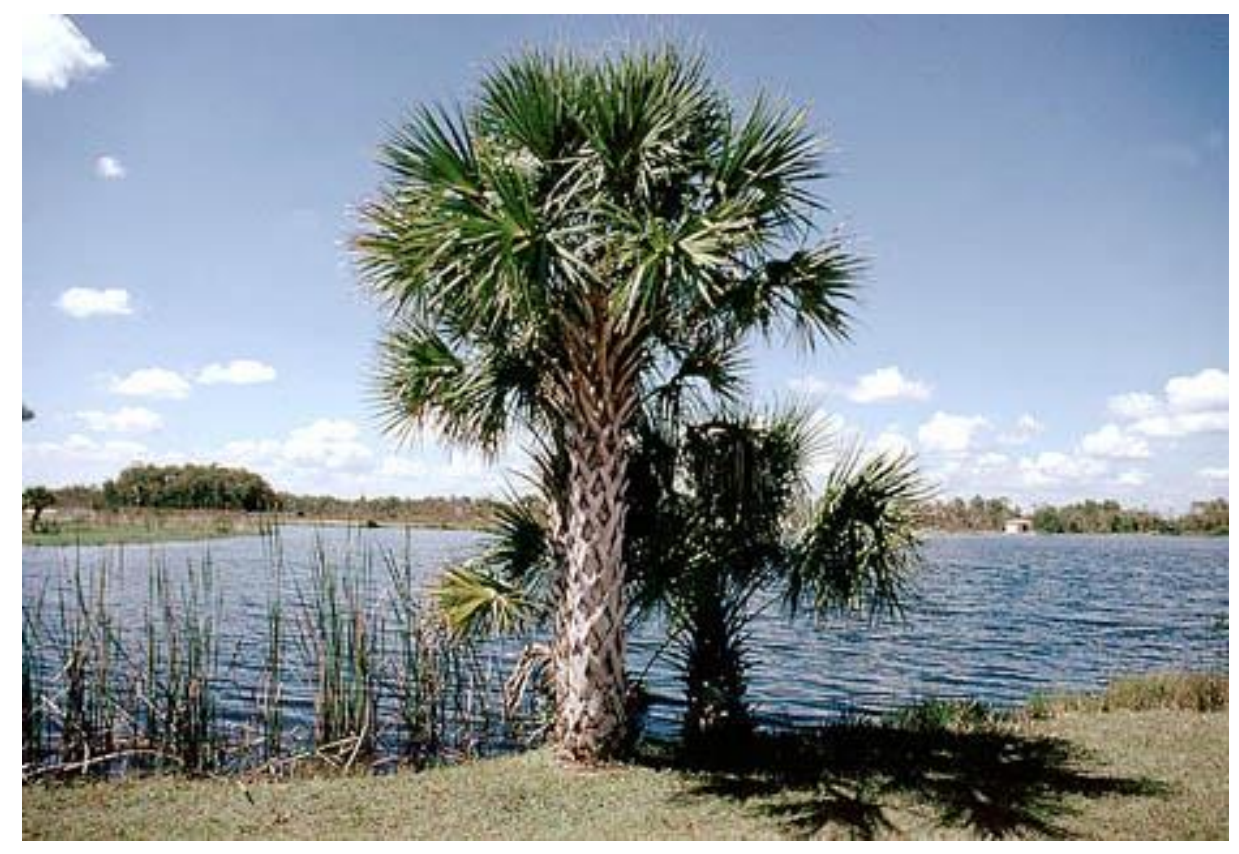

Figure 2: Cabbage palms are so drought and fire-resistant due to their unique spine-like structures that collect leaf debris to insulate from wildfires (Florida eco travel guide, n.d.)

Geographically speaking the entire state of Florida is defined by its water flow. The KissimmeeOkeechobee-Everglades (KOE) watershed is the movement and transportation of water from Orlando down to the Everglades, where it nurtures the state's pride and joy: Everglades National Park. Although nowadays, the natural flow is not as strong due to flood prevention measures, rainfall amount does affect the river discharge (SOFIA, n.d.). Rainfall quantities also have societal implications, in addition to environmental ones. According to the South Florida Water Management District, more than 90 percent of people in northeast and east-central Florida obtain their water from the aquifer (SJRWMD, n.d.). The Floridian Aquifer extends beneath much of Florida in its porous limestone base. When it rains, a process called percolation occurs where the rainwater will drain through the limestone, recharging the aquifer beneath. Fluctuating rainfall amount can lead to drought, which affects fire occurrence and water availability. When drought occurs, it affects lifestyle choice such as water usage for lawns or showers. Also it can have more 
serious consequences. When wildfires occur, and drought breaks out, other adverse weather conditions can occur. In a study by Collins et al. (2009), the deadly smog event that occurred on I-4 in January 2008 was analyzed. The event began as a prescribed wildfire burn, but meteorological and geographical conditions were in favor of chaos, combined with the drought conditions, with five people perished and 38 injured. This is just one of many stories discussed over late-night news that emphasize the danger of wildfires encouraged by dry biomass such as leaves and grasses. Another consequence of drought is the formation of sinkholes. As discussed above, when not enough water percolates through the limestone base of Florida, the water level of the aquifer declines. When the water level drops below the top of the bedrock, a sinkhole can occur due to changes in pressure and overall structure of the cavernous system below the surface (Newton, 1986). The opposite can also occur when it rains too much. Flooding is a frequent problem due to the high water table and urban setting (such as concrete) reducing water penetration into the soil. This urban effect can also have negative impact for the aquifer, as less water can percolate despite the increased rainfall (Gilman, 1985). This can affect many people, as what was seen in the 1928 Okeechobee Hurricane, which was the second deadliest one ever due to the breach of the levees around the lake. It also affects people locally, as seen in June 2016. There was severe flooding in the urban area in Tampa due to heavy rainfall and nonpenetrable materials such as streets. Some communities were shut down for days, with people unable to reach the amenities like grocery stores. Westshore Boulevard, Dale Mabry, and other roads all amassed huge quantities of water (O'Reily, 2016). Thus, careful monitoring of the rainfall and effective urban planning are vital to maximize human lifestyle here in Florida. 


\section{MATHEMATICAL DESCRIPTION AND SOLUTION APPROACH}

To solve the problem presented above, a trapezoidal Riemann's sum and a definite integral generated by polynomial regression are used. The data was attained from NOAA's public access "Global Historical Climatology Network (GHCN) - Daily" database (NOAA, 2016). This data was recorded once a day at midnight for the year of 2016 at the Tampa Bay International Airport weather station. For the purpose of this study, the data was divided by the seasonal changes in 2016 (Calendar Date, n.d.). The meteorological definition of a season was used, as it is the most effective definer over land in the Northern Hemisphere. This divides it up based on the spring equinox, summer solstice, fall equinox, and winter solstice (Trenberth, 1983). Afterwards the total rainfall for the timeframe was recorded, and then graphed (Figure 3). The dark blue line represents the total amount of rainfall during the season, smoothed by Excel 2013. The dotted line on the graph is a $3^{\text {rd }}$ degree polynomial trendline which approximately matches the original dataset. The equation for the trendline was also discovered to give an estimated equation for the graph of the total rainfall of the region defined by the seasons. 


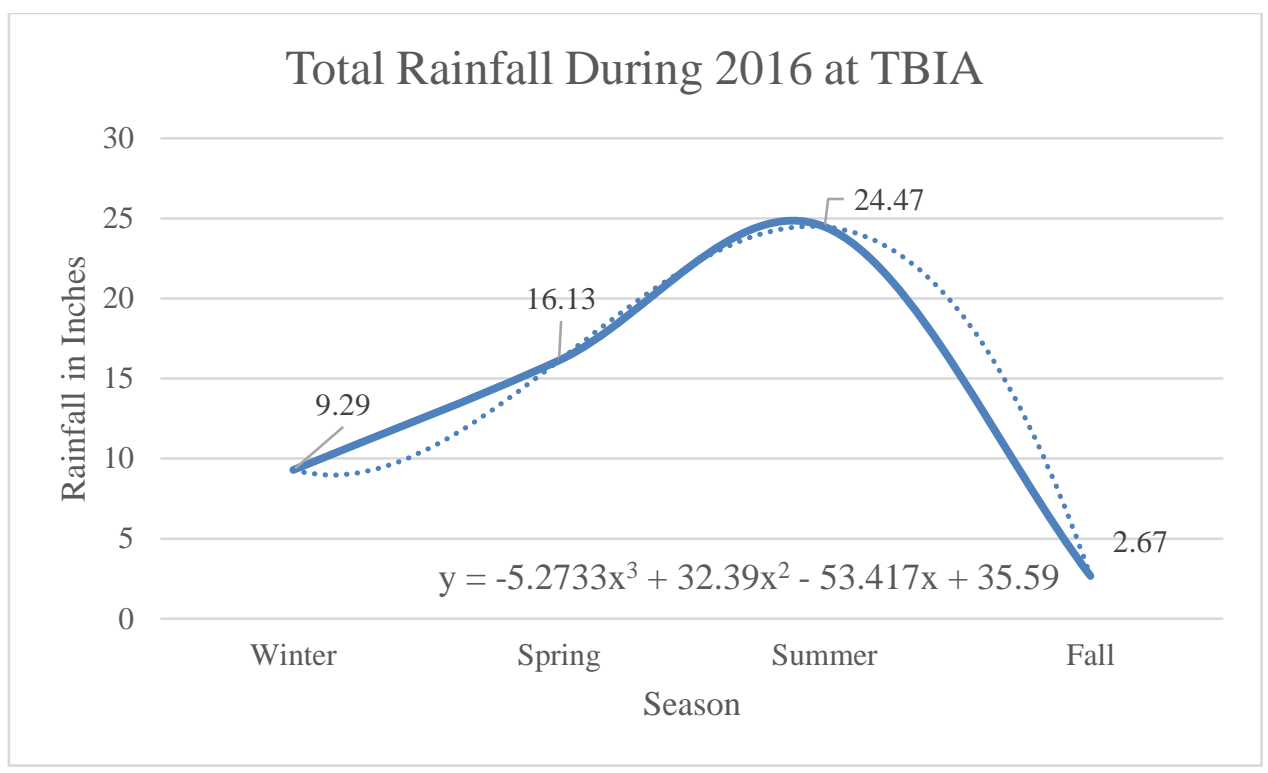

Figure 3: Representation of the total rainfall at the Tampa Bay International Airport (TBIA) during the 2016 seasons.

The seasonal total rainfall is used to get a rough estimate by a trapezoidal Riemann's sum. This form of Riemann's sums fits trapezoidal shapes onto the graph geometrically approximating area. The formula for the trapezoidal Riemann's sum is given in (1) and its value in our case is given in (2):

$$
\begin{aligned}
& \text { Area } \approx \Delta x\left[\left(\frac{1}{2}\right)\left(y_{1}+y_{2}\right)+\left(\frac{1}{2}\right)\left(y_{2}+y_{3}\right)+\ldots+\left(\frac{1}{2}\right)\left(y_{n-1}+y_{n}\right)\right], \\
& \left(\frac{1}{2}\right)(9.29+16.13)+\left(\frac{1}{2}\right)(16.13+24.47)+\left(\frac{1}{2}\right)(24.47+2.67) \approx 46.58,
\end{aligned}
$$

where $\Delta x=1$ and three trapezoids are used.

To determine a more accurate estimate we use the corresponding polynomial integral. With the equation of the $3^{\text {rd }}$ degree polynomial trendline fitted to the above graph (Figure 3 ) the following definite integral was constructed to determine the total amount of rainfall in the area and compare it to the total rainfall recorded by NOAA (see (3-5)):

Total rainfall for $2016 \approx \int_{1}^{4}\left(-5.2733 x^{3}+32.39 x^{2}-53.417 x+35.59\right) d x$ 
Total rainfall $\approx \frac{-5.2733 x^{4}}{4}+\frac{32.39 x^{3}}{3}-\frac{53.417 x^{2}}{2}+35.59 x$ from 1 to 4

Total rainfall for $2016 \approx 50.1596$ inches of rain

\section{DISCUSSION}

The recorded value for the total rainfall at Tampa Bay International Airport in 2016 was recorded by NOAA as 52.59 inches. As shown in the above equations and graphs, the polynomial integral gives a value of 50.16 inches of rain, and the trapezoidal Riemann's sum has a value of 46.58 inches of rain. The percent error, calculated as

$$
\text { percent error: } \frac{\mid \text { experimental value-theoretical value } \mid}{\text { theoretical value }} \times 100 \%
$$

shows that the integral gives a $4.62 \%$ error, and the Riemann's sum gives a $11.43 \%$ error. Both were the underestimates of the true value, since they yielded to a lower value for the total inches of rain than it was truly recorded by NOAA. The objectives for this project were met since both the trapezoidal Riemann's sum and polynomial integral were used to approximate the rainfall in Tampa. Overall, the results from the year match up to the expected precipitation values as defined by the Köppen climate classification system. According to Encyclopedia Brittanica, a humid subtropical climate ( $\mathrm{Cfa}$ ) has an average precipitation amount of 39 inches, but can fall anywhere in the range of 30 to 79 inches of precipitation annually. There was also a recorded seasonal summer spike in precipitation, which is characteristic for a humid subtropical classification. Thus, this year does have rainfall slightly higher than usual. Also, the hypothesis that the definite integral generated by polynomial regression would better predict was shown to be correct. 


\section{CONCLUSION AND RECOMMENDATIONS}

It can be concluded that the definite integral generated by polynomial regression is most accurate in predicting rainfall from the graph. Both the polynomial integral and trapezoidal Riemann's sum underestimated the total rainfall, but overall the estimates were fairly accurate as shown via the percent error. The representation of the data and the total recorded rainfall matches with the previously established Köppen classification system. In the future, if one wants to use calculus to determine rainfall from an area, polynomial integrals are shown to be more effective predictors of the actual amount. Future research could center on using more detailed data, such as hourly recordings, or designing a computer algorithm to predict future rainfall based on the proper $n$-th degree polynomial trendline $(n \geq 3)$.

\section{NOMENCLATURE}

\begin{tabular}{|l|l|}
\hline In. & Inches \\
\hline Cfa & Humid Subtropical Climate type \\
\hline
\end{tabular}

\section{REFERENCES}

"Cabbage Palm or Sabal Palmetto." Florida eco travel guide. N.p., n.d. Web. 04 May 2017.

"Climate Classification and Climatic Regions of the World." Fundamentals eBook. PhysicalGeography.net, n.d. Web. 04 May 2017

Collins, Jennifer M., Alicia N. Williams, Charles H. Paxton, Richard J. Davis, and Nicholas M. Petro. "Geographical, Meteorological, and Climatological Conditions Surrounding 
the 2008 Interstate-4 Disaster in Florida." Papers of the Applied Geography Conferences (2009): 153-62. Web. 6 May 2017.

The Editors of Encyclopædia Britannica. "Humid Subtropical Climate." Encyclopæedia Britannica. Encyclopædia Britannica, inc., n.d. Web. 06 May 2017.

"Florida’s Aquifers." Water Supply. St. Johns River Water Management District , n.d. Web. 04 May 2017.

"Florida Climate." WeatherSTEM. N.p., n.d. Web. 04 May 2017. NOAA. National Centers for Environmental Prediction. N.p., n.d. Web. 4 May 2017. <https://www.ncdc.noaa.gov/data-access>.

Gilman, Harold F., and Philip D. Jolly. "Urbanization and Hydrologic Change in Northwest Hillsborough County, Florida ." Th Florida Geographer (1985): n. pag. Web. 6 May 2017.

Newton, J. G. "Natural and Induced Sinkhole Development in the Eastern United States." U.S. Geological Survey (1986): n. pag. Web.

O'Reilly, Sean. "TRAFFIC ALERT: Westshore Boulevard in Tampa closing on Wednesday to drivers for up to three months." WFTS. N.p., 11 May 2016. Web. 06 May 2017. "The South Florida Environment: A Region Under Stress." SOFIA. USGS, n.d. Web. 04 May 2017.

"2016 Seasons Calendar." Calendar Date. N.p., n.d. Web. 04 May 2017.

Trenberth, Kevin E. "What are the Seasons?" Bulletin of the American Meteorological Society (1983): n. pag. Web. 6 May 2017. 


\section{$\underline{\text { Appendices }}$}

Table 1 (below): Precipitation dataset extracted from NOAA's Tampa Bay International Airport location, and seasonal totals obtained from the daily values

\begin{tabular}{|c|c|c|c|}
\hline DATE & $\begin{array}{l}\text { RAINFALL } \\
\text { (inches) }\end{array}$ & $\begin{array}{l}\text { Total Rai } \\
\text { Season (i }\end{array}$ & $\begin{array}{l}\text { II by } \\
\text { hes) }\end{array}$ \\
\hline $1 / 1 / 2016$ & 0.00 & Winter & 9.29 \\
\hline $1 / 2 / 2016$ & 0.00 & Spring & 16.13 \\
\hline $1 / 3 / 2016$ & 0.18 & Summer & 24.47 \\
\hline $1 / 4 / 2016$ & 0.00 & Fall & 2.67 \\
\hline $1 / 5 / 2016$ & 0.00 & & \\
\hline $1 / 6 / 2016$ & 0.00 & & \\
\hline $1 / 7 / 2016$ & 0.00 & & \\
\hline $1 / 8 / 2016$ & 0.54 & & \\
\hline $1 / 9 / 2016$ & 0.65 & & \\
\hline $1 / 10 / 2016$ & 0.00 & & \\
\hline $1 / 11 / 2016$ & 0.00 & & \\
\hline $1 / 12 / 2016$ & 0.00 & & \\
\hline $1 / 13 / 2016$ & 0.00 & & \\
\hline $1 / 14 / 2016$ & 0.02 & & \\
\hline $1 / 15 / 2016$ & 0.54 & & \\
\hline $1 / 16 / 2016$ & 0.03 & & \\
\hline $1 / 17 / 2016$ & 1.01 & & \\
\hline $1 / 18 / 2016$ & 0.00 & & \\
\hline $1 / 19 / 2016$ & 0.00 & & \\
\hline $1 / 20 / 2016$ & 0.00 & & \\
\hline $1 / 21 / 2016$ & 0.00 & & \\
\hline $1 / 22 / 2016$ & 0.56 & & \\
\hline $1 / 23 / 2016$ & 0.10 & & \\
\hline $1 / 24 / 2016$ & 0.00 & & \\
\hline $1 / 25 / 2016$ & 0.00 & & \\
\hline $1 / 26 / 2016$ & 0.01 & & \\
\hline $1 / 27 / 2016$ & 1.51 & & \\
\hline $1 / 28 / 2016$ & 1.03 & & \\
\hline $1 / 29 / 2016$ & 0.00 & & \\
\hline $1 / 30 / 2016$ & 0.00 & & \\
\hline $1 / 31 / 2016$ & 0.00 & & \\
\hline $2 / 1 / 2016$ & 0.00 & & \\
\hline $2 / 2 / 2016$ & 0.00 & & \\
\hline $2 / 3 / 2016$ & 0.00 & & \\
\hline $2 / 4 / 2016$ & 1.05 & & \\
\hline
\end{tabular}




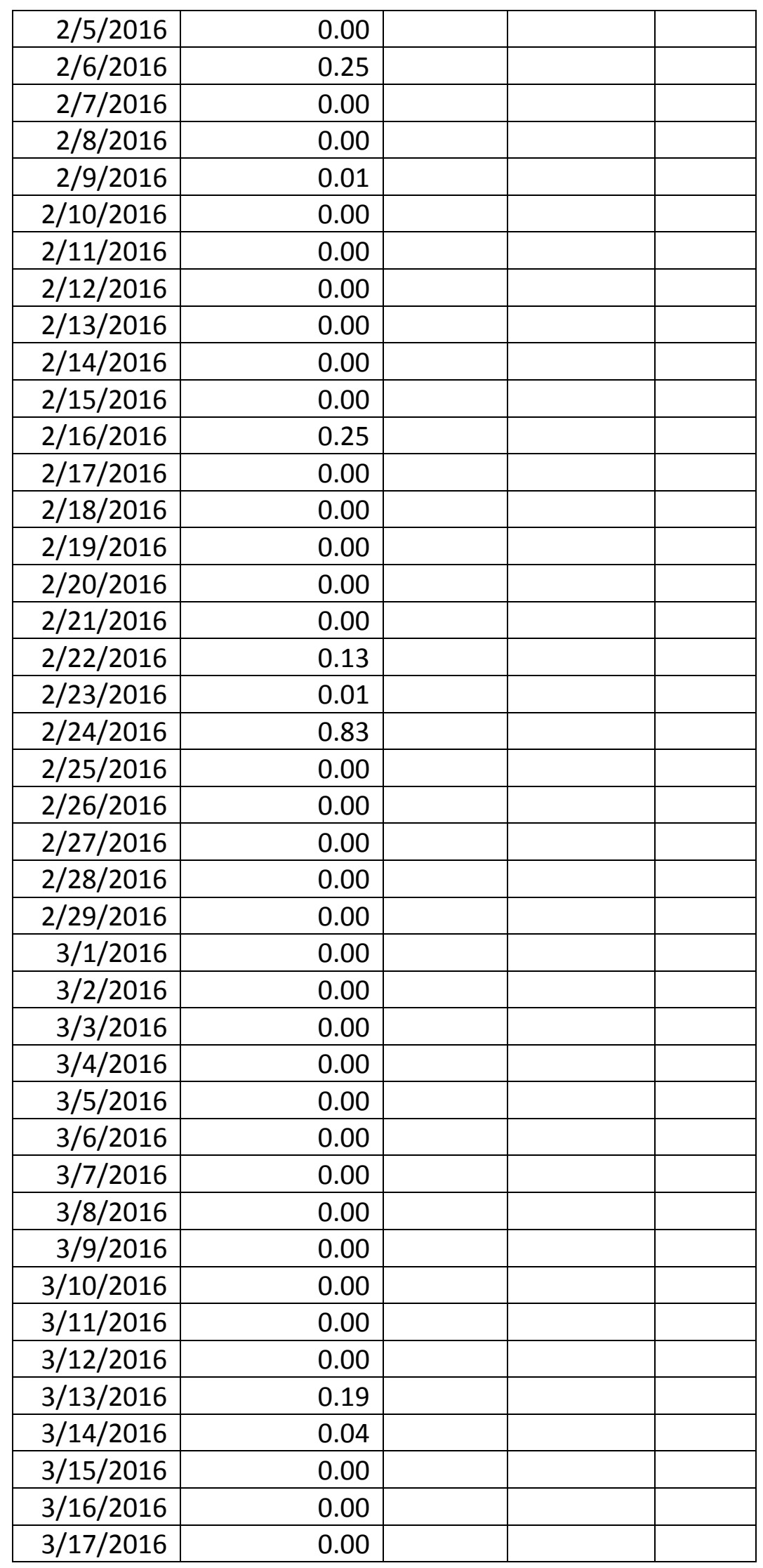




\begin{tabular}{|r|r|l|l|l|}
\hline $3 / 18 / 2016$ & 0.00 & & & \\
\hline $3 / 19 / 2016$ & 0.33 & & & \\
\hline $3 / 20 / 2016$ & 0.00 & & & \\
\hline $3 / 21 / 2016$ & 0.00 & & & \\
\hline $3 / 22 / 2016$ & 0.00 & & & \\
\hline $3 / 23 / 2016$ & 0.00 & & & \\
\hline $3 / 24 / 2016$ & 0.07 & & & \\
\hline $3 / 25 / 2016$ & 0.72 & & & \\
\hline $3 / 26 / 2016$ & 0.00 & & & \\
\hline $3 / 27 / 2016$ & 0.00 & & & \\
\hline $3 / 28 / 2016$ & 0.03 & & & \\
\hline $3 / 29 / 2016$ & 0.38 & & & \\
\hline $3 / 30 / 2016$ & 0.00 & & & \\
\hline $3 / 31 / 2016$ & 0.00 & & & \\
\hline $4 / 1 / 2016$ & 0.00 & & & \\
\hline $4 / 2 / 2016$ & 0.68 & & & \\
\hline $4 / 3 / 2016$ & 0.00 & & & \\
\hline $4 / 4 / 2016$ & 0.00 & & & \\
\hline $4 / 5 / 2016$ & 0.00 & & & \\
\hline $4 / 6 / 2016$ & 0.00 & & & \\
\hline $4 / 7 / 2016$ & 0.70 & & & \\
\hline $4 / 8 / 2016$ & 0.00 & & & \\
\hline $4 / 9 / 2016$ & 0.00 & & & \\
\hline $4 / 10 / 2016$ & 0.00 & & & \\
\hline $4 / 11 / 2016$ & 0.00 & & & \\
\hline $4 / 12 / 2016$ & 0.00 & & & \\
\hline $4 / 13 / 2016$ & 0.00 & & & \\
\hline $4 / 14 / 2016$ & 0.00 & & & \\
\hline $4 / 15 / 2016$ & 0.06 & & & \\
\hline $4 / 16 / 2016$ & 0.00 & & & \\
\hline $4 / 17 / 2016$ & 0.00 & & & \\
\hline $4 / 18 / 2016$ & 0.00 & & & \\
\hline $4 / 19 / 2016$ & 0.00 & & & \\
\hline $4 / 20 / 2016$ & 0.00 & & & \\
\hline $4 / 21 / 2016$ & 0.00 & & & \\
\hline $4 / 22 / 2016$ & 0.07 & & & \\
\hline $4 / 23 / 2016$ & 0.00 & & & \\
\hline $4 / 24 / 2016$ & 0.03 & & & \\
\hline $4 / 25 / 2016$ & 0.13 & & & \\
\hline $4 / 26 / 2016$ & 0.00 & & & \\
\hline $4 / 27 / 2016$ & 0.00 & & & \\
\hline $4 / 28 / 2016$ & & & & \\
\hline & 0.00 & & \\
\hline
\end{tabular}




\begin{tabular}{|r|r|l|l|l|}
\hline $4 / 29 / 2016$ & 0.00 & & & \\
\hline $4 / 30 / 2016$ & 0.00 & & & \\
\hline $5 / 1 / 2016$ & 0.00 & & & \\
\hline $5 / 2 / 2016$ & 0.00 & & & \\
\hline $5 / 3 / 2016$ & 0.00 & & & \\
\hline $5 / 4 / 2016$ & 2.79 & & & \\
\hline $5 / 5 / 2016$ & 0.00 & & & \\
\hline $5 / 6 / 2016$ & 0.00 & & & \\
\hline $5 / 7 / 2016$ & 0.00 & & & \\
\hline $5 / 8 / 2016$ & 0.00 & & & \\
\hline $5 / 9 / 2016$ & 0.00 & & & \\
\hline $5 / 10 / 2016$ & 0.00 & & & \\
\hline $5 / 11 / 2016$ & 0.00 & & & \\
\hline $5 / 12 / 2016$ & 0.00 & & & \\
\hline $5 / 13 / 2016$ & 0.00 & & & \\
\hline $5 / 14 / 2016$ & 0.00 & & & \\
\hline $5 / 15 / 2016$ & 0.00 & & & \\
\hline $5 / 16 / 2016$ & 0.00 & & & \\
\hline $5 / 17 / 2016$ & 0.40 & & & \\
\hline $5 / 18 / 2016$ & 0.00 & & & \\
\hline $5 / 19 / 2016$ & 0.00 & & & \\
\hline $5 / 20 / 2016$ & 0.57 & & & \\
\hline $5 / 21 / 2016$ & 0.00 & & & \\
\hline $5 / 22 / 2016$ & 0.00 & & & \\
\hline $5 / 23 / 2016$ & 0.00 & & & \\
\hline $5 / 24 / 2016$ & 0.00 & & & \\
\hline $5 / 25 / 2016$ & 0.00 & & & \\
\hline $5 / 26 / 2016$ & 0.00 & & & \\
\hline $5 / 27 / 2016$ & 0.00 & & & \\
\hline $5 / 28 / 2016$ & 0.00 & & & \\
\hline $5 / 29 / 2016$ & 0.00 & & & \\
\hline $5 / 30 / 2016$ & 0.00 & & & \\
\hline $5 / 31 / 2016$ & 0.00 & & & \\
\hline $6 / 1 / 2016$ & 0.02 & & & \\
\hline $6 / 2 / 2016$ & 0.04 & & & \\
\hline $6 / 3 / 2016$ & 0.00 & & & \\
\hline $6 / 4 / 2016$ & 0.18 & & & \\
\hline $6 / 5 / 2016$ & 0.77 & & & \\
\hline $6 / 6 / 2016$ & 2.76 & & & \\
\hline $6 / 7 / 2016$ & 0.78 & & & \\
\hline $6 / 8 / 2016$ & 0.10 & & & \\
\hline $6 / 9 / 2016$ & & & \\
\hline
\end{tabular}




\begin{tabular}{|r|r|l|l|l|}
\hline $6 / 10 / 2016$ & 0.52 & & \\
\hline $6 / 11 / 2016$ & 0.21 & & & \\
\hline $6 / 12 / 2016$ & 1.28 & & & \\
\hline $6 / 13 / 2016$ & 0.52 & & & \\
\hline $6 / 14 / 2016$ & 0.00 & & & \\
\hline $6 / 15 / 2016$ & 0.00 & & & \\
\hline $6 / 16 / 2016$ & 0.00 & & & \\
\hline $6 / 17 / 2016$ & 0.02 & & & \\
\hline $6 / 18 / 2016$ & 0.05 & & & \\
\hline $6 / 19 / 2016$ & 0.00 & & & \\
\hline $6 / 20 / 2016$ & 0.00 & & & \\
\hline $6 / 21 / 2016$ & 0.00 & & & \\
\hline $6 / 22 / 2016$ & 0.00 & & & \\
\hline $6 / 23 / 2016$ & 0.00 & & & \\
\hline $6 / 24 / 2016$ & 0.00 & & & \\
\hline $6 / 25 / 2016$ & 0.54 & & & \\
\hline $6 / 26 / 2016$ & 1.20 & & & \\
\hline $6 / 27 / 2016$ & 0.07 & & & \\
\hline $6 / 28 / 2016$ & 0.00 & & & \\
\hline $6 / 29 / 2016$ & 0.00 & & & \\
\hline $6 / 30 / 2016$ & 0.17 & & & \\
\hline $7 / 1 / 2016$ & 0.00 & & & \\
\hline $7 / 2 / 2016$ & 0.30 & & & \\
\hline $7 / 3 / 2016$ & 0.00 & & & \\
\hline $7 / 4 / 2016$ & 0.00 & & & \\
\hline $7 / 5 / 2016$ & 0.00 & & & \\
\hline $7 / 6 / 2016$ & 0.00 & & & \\
\hline $7 / 7 / 2016$ & 0.00 & & & \\
\hline $7 / 8 / 2016$ & 0.00 & & & \\
\hline $7 / 9 / 2016$ & 0.00 & & & \\
\hline $7 / 10 / 2016$ & 0.00 & & & \\
\hline $7 / 11 / 2016$ & 0.35 & & & \\
\hline $7 / 12 / 2016$ & 0.52 & & & \\
\hline $7 / 13 / 2016$ & 0.00 & & & \\
\hline $7 / 14 / 2016$ & 0.07 & & & \\
\hline $7 / 15 / 2016$ & 0.01 & & & \\
\hline $7 / 16 / 2016$ & 0.00 & & & \\
\hline $7 / 17 / 2016$ & 0.11 & & & \\
\hline $7 / 18 / 2016$ & 0.00 & & & \\
\hline $7 / 19 / 2016$ & 0.00 & & & \\
\hline $7 / 20 / 2016$ & 0.04 & & & \\
\hline $7 / 21 / 2016$ & & & \\
\hline
\end{tabular}




\begin{tabular}{|r|r|l|l|l|}
\hline $7 / 22 / 2016$ & 0.52 & & & \\
\hline $7 / 23 / 2016$ & 0.00 & & & \\
\hline $7 / 24 / 2016$ & 1.31 & & & \\
\hline $7 / 25 / 2016$ & 0.00 & & & \\
\hline $7 / 26 / 2016$ & 1.34 & & & \\
\hline $7 / 27 / 2016$ & 0.36 & & & \\
\hline $7 / 28 / 2016$ & 0.07 & & & \\
\hline $7 / 29 / 2016$ & 0.03 & & & \\
\hline $7 / 30 / 2016$ & 0.05 & & & \\
\hline $7 / 31 / 2016$ & 1.26 & & & \\
\hline $8 / 1 / 2016$ & 0.00 & & & \\
\hline $8 / 2 / 2016$ & 0.00 & & & \\
\hline $8 / 3 / 2016$ & 0.00 & & & \\
\hline $8 / 4 / 2016$ & 1.91 & & & \\
\hline $8 / 5 / 2016$ & 0.00 & & & \\
\hline $8 / 6 / 2016$ & 0.00 & & & \\
\hline $8 / 7 / 2016$ & 0.40 & & & \\
\hline $8 / 8 / 2016$ & 1.98 & & & \\
\hline $8 / 9 / 2016$ & 0.39 & & & \\
\hline $8 / 10 / 2016$ & 0.00 & & & \\
\hline $8 / 11 / 2016$ & 0.09 & & & \\
\hline $8 / 12 / 2016$ & 0.01 & & & \\
\hline $8 / 13 / 2016$ & 0.00 & & & \\
\hline $8 / 14 / 2016$ & 0.00 & & & \\
\hline $8 / 15 / 2016$ & 0.00 & & & \\
\hline $8 / 16 / 2016$ & 0.79 & & & \\
\hline $8 / 17 / 2016$ & 0.29 & & & \\
\hline $8 / 18 / 2016$ & 0.08 & & & \\
\hline $8 / 19 / 2016$ & 0.00 & & & \\
\hline $8 / 20 / 2016$ & 0.00 & & & \\
\hline $8 / 21 / 2016$ & 0.00 & & & \\
\hline $8 / 22 / 2016$ & 0.00 & & & \\
\hline $8 / 23 / 2016$ & 0.52 & & & \\
\hline $8 / 24 / 2016$ & 0.01 & & & \\
\hline $8 / 25 / 2016$ & 0.00 & & & \\
\hline $8 / 26 / 2016$ & 0.39 & & & \\
\hline $8 / 27 / 2016$ & 0.06 & & & \\
\hline $8 / 28 / 2016$ & 0.04 & & & \\
\hline $8 / 29 / 2016$ & 1.27 & & & \\
\hline $8 / 30 / 2016$ & 0.44 & & & \\
\hline $8 / 31 / 2016$ & 0.04 & & & \\
\hline $9 / 1 / 2016$ & & & & \\
\hline & & & \\
\hline
\end{tabular}




\begin{tabular}{|r|r|l|l|l|}
\hline $9 / 2 / 2016$ & 1.23 & & \\
\hline $9 / 3 / 2016$ & 0.52 & & & \\
\hline $9 / 4 / 2016$ & 0.00 & & & \\
\hline $9 / 5 / 2016$ & 0.46 & & & \\
\hline $9 / 6 / 2016$ & 0.00 & & & \\
\hline $9 / 7 / 2016$ & 0.00 & & & \\
\hline $9 / 8 / 2016$ & 0.00 & & & \\
\hline $9 / 9 / 2016$ & 0.00 & & & \\
\hline $9 / 10 / 2016$ & 0.00 & & & \\
\hline $9 / 11 / 2016$ & 0.00 & & & \\
\hline $9 / 12 / 2016$ & 0.00 & & & \\
\hline $9 / 13 / 2016$ & 0.02 & & & \\
\hline $9 / 14 / 2016$ & 0.00 & & & \\
\hline $9 / 15 / 2016$ & 0.00 & & & \\
\hline $9 / 16 / 2016$ & 0.00 & & & \\
\hline $9 / 17 / 2016$ & 0.00 & & & \\
\hline $9 / 18 / 2016$ & 0.00 & & & \\
\hline $9 / 19 / 2016$ & 0.00 & & & \\
\hline $9 / 20 / 2016$ & 0.02 & & & \\
\hline $9 / 21 / 2016$ & 0.00 & & & \\
\hline $9 / 22 / 2016$ & 0.00 & & & \\
\hline $9 / 23 / 2016$ & 0.00 & & & \\
\hline $9 / 24 / 2016$ & 0.00 & & & \\
\hline $9 / 25 / 2016$ & 0.26 & & & \\
\hline $9 / 26 / 2016$ & 0.00 & & & \\
\hline $9 / 27 / 2016$ & 0.00 & & & \\
\hline $9 / 28 / 2016$ & 0.28 & & & \\
\hline $9 / 29 / 2016$ & 0.09 & & & \\
\hline $9 / 30 / 2016$ & 0.07 & & & \\
\hline $10 / 1 / 2016$ & 0.02 & & & \\
\hline $10 / 2 / 2016$ & 0.70 & & & \\
\hline $10 / 3 / 2016$ & 0.20 & & & \\
\hline $10 / 4 / 2016$ & 0.01 & & & \\
\hline $10 / 5 / 2016$ & 0.00 & & & \\
\hline $10 / 6 / 2016$ & 0.25 & & & \\
\hline $10 / 7 / 2016$ & 0.13 & & & \\
\hline $10 / 8 / 2016$ & 0.04 & & & \\
\hline $10 / 9 / 2016$ & 0.00 & & & \\
\hline $10 / 10 / 2016$ & 0.00 & & & \\
\hline $10 / 11 / 2016$ & 0.00 & & & \\
\hline $10 / 12 / 2016$ & 0.00 & & & \\
\hline $10 / 13 / 2016$ & & & & \\
\hline & & & \\
\hline
\end{tabular}




\begin{tabular}{|c|l|l|l|l|}
\hline $10 / 14 / 2016$ & 0.00 & & & \\
\hline $10 / 15 / 2016$ & 0.20 & & & \\
\hline $10 / 16 / 2016$ & 0.00 & & & \\
\hline $10 / 17 / 2016$ & 0.00 & & & \\
\hline $10 / 18 / 2016$ & 0.00 & & & \\
\hline $10 / 19 / 2016$ & 0.00 & & & \\
\hline $10 / 20 / 2016$ & 0.00 & & & \\
\hline $10 / 21 / 2016$ & 0.00 & & & \\
\hline $10 / 22 / 2016$ & 0.00 & & & \\
\hline $10 / 23 / 2016$ & 0.00 & & & \\
\hline $10 / 24 / 2016$ & 0.00 & & & \\
\hline $10 / 25 / 2016$ & 0.00 & & & \\
\hline $10 / 26 / 2016$ & 0.00 & & & \\
\hline $10 / 27 / 2016$ & 0.00 & & & \\
\hline $10 / 28 / 2016$ & 0.00 & & & \\
\hline $10 / 29 / 2016$ & 0.00 & & & \\
\hline $10 / 30 / 2016$ & 0.00 & & & \\
\hline $10 / 31 / 2016$ & 0.00 & & & \\
\hline $11 / 1 / 2016$ & 0.00 & & & \\
\hline $11 / 2 / 2016$ & 0.00 & & & \\
\hline $11 / 3 / 2016$ & 0.00 & & & \\
\hline $11 / 4 / 2016$ & 0.00 & & & \\
\hline $11 / 5 / 2016$ & 0.00 & & & \\
\hline $11 / 6 / 2016$ & 0.00 & & & \\
\hline $11 / 7 / 2016$ & 0.00 & & & \\
\hline $11 / 8 / 2016$ & 0.00 & & & \\
\hline $11 / 9 / 2016$ & 0.01 & & & \\
\hline $11 / 10 / 2016$ & 0.00 & & & \\
\hline $11 / 11 / 2016$ & 0.00 & & & \\
\hline $11 / 12 / 2016$ & 0.00 & & & \\
\hline $11 / 13 / 2016$ & 0.00 & & & \\
\hline $11 / 14 / 2016$ & 0.00 & & & \\
\hline $11 / 15 / 2016$ & 0.00 & & & \\
\hline $11 / 16 / 2016$ & 0.00 & & & \\
\hline $11 / 17 / 2016$ & 0.00 & & & \\
\hline $11 / 18 / 2016$ & 0.00 & & & \\
\hline $11 / 19 / 2016$ & 0.00 & & & \\
\hline $11 / 20 / 2016$ & 0.00 & & & \\
\hline $11 / 21 / 2016$ & 0.00 & & & \\
\hline $11 / 22 / 2016$ & 0.00 & & & \\
\hline $11 / 23 / 2016$ & 0.00 & & & \\
\hline $11 / 24 / 2016$ & & & \\
\hline
\end{tabular}




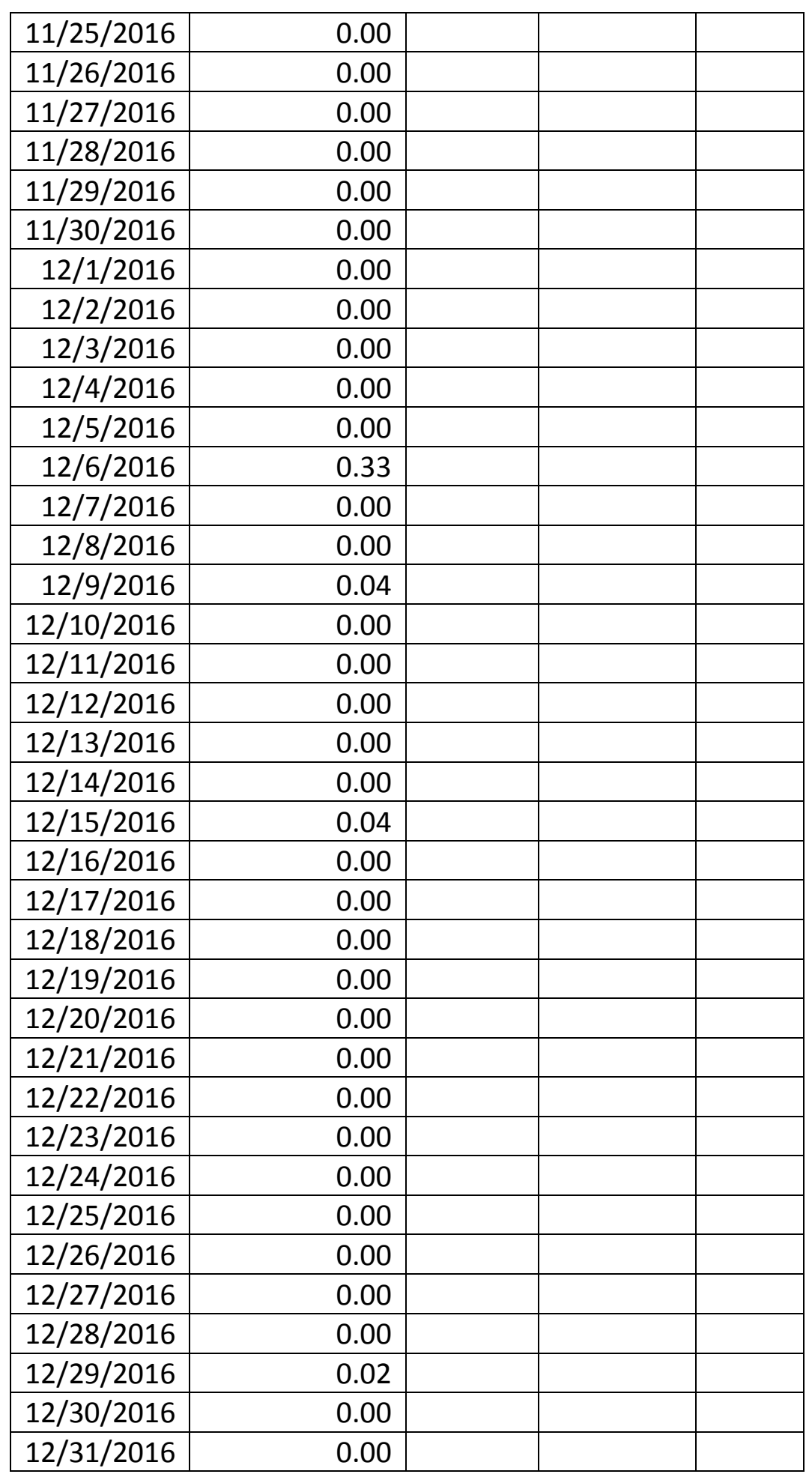

